Archives de sciences sociales des religions

159 | juillet-septembre 2012

Durkheim : Les formes élémentaires de la vie religieuse (1912)

\title{
Les recensions des Formes du vivant de leur auteur (1912-1917)
}

Document

\section{Stéphane Baciocchi}

\section{(2) OpenEdition \\ Journals}

Édition électronique

URL : http://journals.openedition.org/assr/24225

DOI : $10.4000 /$ assr.24225

ISSN : $1777-5825$

Éditeur

Éditions de l'EHESS

Édition imprimée

Date de publication : 30 septembre 2012

Pagination : 17-27

ISBN : 978-2-7132-2330-3

ISSN : 0335-5985

\section{Référence électronique}

Stéphane Baciocchi, «Les recensions des Formes du vivant de leur auteur (1912-1917) 》, Archives de sciences sociales des religions [En ligne], 159 | juillet-septembre 2012, mis en ligne le 26 novembre 2016, consulté le 19 avril 2019. URL : http://journals.openedition.org/assr/24225; DOI : 10.4000/ assr.24225

(C) Archives de sciences sociales des religions 


\section{Stéphane Baciocchi}

\section{Document}

\section{Les recensions des Formes du vivant de leur auteur (1912-1917)}

La présente bibliographie vise à décrire, en amont des héritages et des oublis dont procède la postérité des Formes élémentaires, ce que fut l'actualité de l'ouvrage, du vivant de son auteur. Elle vaut à la fois comme document et comme instrument de recherche sur la première réception du «dernier ouvrage de Durkheim ", c'est-à-dire, suivant le point de vue des contemporains et l'horizon des recensions considérées, le plus récent en date. Comme instrument de recherche, cette bibliographie propose simplement d'élargir le terrain documentaire sur lequel se fondent les études spécialisées consacrées aux réceptions de l'ouvrage dans tels ou tels espaces de discussion, qu'ils soient disciplinaires, régionaux ou thématiques. Pris ensemble, depuis la thèse d'Arnold Van Gennep (1920) et le mémoire pionnier d'Imogen Seger (1957), les travaux académiques de référence sur la réception des Formes ont identifié et circonscrit un corpus de 20 comptes rendus publiés entre 1912 et $1917^{1}$ : nous en ajoutons 54 . Cet élargissement du corpus de référence résulte d'une investigation de première main sur les sources proprement éditoriales de l'ouvrage, notamment sa traduction et sa promotion, et, d'autre part, sur les repérages indigènes des espaces et domaines à l'intérieur desquels la circulation du livre de Durkheim était attestée (Fargues, 1913 ; Fonsegrive, 1915 ; Gehlke, 1915 ; Jordan, 1915 ; Coe, 1916 ; Simon, 1918 et Van Gennep, 1920). Dans ce cadre, pour la période considérée, nous avons systématiquement examiné les publications bibliographiques internationales ${ }^{2}$ ainsi que les tables et index des principaux périodiques spécialisés ou généralistes dont l'accessibilité tend à se simplifier et à se décloisonner grâce aux instruments numériques contemporains ${ }^{3}$.

1. Lukes, 1988 [1973] ; Pickering, 1975, 1984 et 2008; Nandan, 1977 ; Goddijn, 1983 ; Rawls, 1997 ; Allen, Pickering et Watts-Miller, 1998 ; Morrison, 2001 ; Fournier, 2007 et Pizarro Noël, 2009.

2. Notamment les volumes 16 à 30 (1912-1917) de l'importante Bibliographie der Rezensionen mit Einschluß von Referaten und Selbstanzeigen publiée en supplément de l'Internationale Bibliographie der Zeitschriftenliteratur (Leipzig), le Polybiblion, revue bibliographique universelle (Paris) et The Book Review Digest (White Plains/New York).

3. À la date de notre dernier pointage (août 2012), 51 des 74 textes identifiés sont directement repérables et accessibles en ligne sous une forme digitale, soit librement (Gallica, Internet Archive...), soit via un abonnement commercial (Jstor, ProQuest, Gale, Wiley...). L'accessibilité 
Notre bibliographie reste donc ouverte à des ajouts qui viendront sans doute de recherches plus approfondies dans les archives digitales en expansion, notamment celles de la presse quotidienne et des petites revues périphériques. L'absence de recensions en langue espagnole, portugaise ou russe s'explique par notre incompétence linguistique.

Comme document, notre bibliographie atteste que la publication annoncée en mai 1912 sous le titre Les Formes primitives de la Vie religieuse ne passa pas inaperçue. Imprimé à 1650 exemplaires et disponible à partir du 14-15 juin suivant ${ }^{4}$, le gros volume in-octavo et sa traduction anglo-américaine de septembre 1915 (1 000 copies) furent recensés par une multiplicité de journaux et revues publiés dans plusieurs des grandes capitales culturelles et académiques occidentales : en premier lieu à Paris et Londres, à New York, Leipzig, Chicago, Édimbourg, Philadelphie, Berlin et Dublin, mais aussi à Francfort, Rome, Bologne, Gènes, Genève, Lausanne, Kain, Lviv, Lancaster, Worcester, Melbourne et Bordeaux. La liste des recensions révèle que la carrière éditoriale de l'ouvrage a suivi une trame chronologique où l'on distingue nettement deux moments, de part et d'autre de la rupture ouverte par le déclenchement de la Première Guerre mondiale (Graph. 1).

La constitution antagonique des deux grandes alliances européennes mis sans doute fin au projet d'une traduction allemande de l'ouvrage tandis que le jeune étudiant américain Joseph Ward Swain pouvait proposer, en octobre 1914, une traduction anglaise qu'il réalisa durant la première année de la guerre, dans le cours de ses circulations entre Paris, Londres, Zurich et New York ${ }^{5}$. Cette traduction facilita l'internationalisation de l'ouvrage. Elle vint relancer, et dans une certaine mesure, délocaliser, notamment du côté américain, les premiers espaces de discussions nouées principalement durant le printemps 1913 sur la scène philosophique et confessionnelle parisienne. Les suggestions de titres avancés par Durkheim lui-même pour la traduction anglophone de son ouvrage indiquent explicitement ce changement d'horizon : "Totemism: a Study of the Elementary Religious Life » puis « Totemism: a Study in Religious Sociology » 6 .

et le décloisonnement sont donc très partiels et particulièrement piégeurs si l'on considère que des textes aussi importants pour notre enquête que celui d'Andrew Lang, Isaac Benrubi, Madeleine Matisse, Goblet d'Alviella ou encore Paul Onésisme Habert sont passés à travers les mailles des vastes campagnes industrielles de numérisation.

4. Archives nationales (Paris) - F18* (IX) 310, Dépôt légal des ouvrages littéraires (écrits non périodiques), département de l'Eure, le 17 juillet 1912, no 213 : "Les Formes élémentaires, de Durkheim, Hérissey, à Évreux. 41 f. d'imprimerie, 1650 exemplaires déclarés. » ; Bulletin des publications nouvelles de la librairie Félix Alcan (Félix Alcan et R. Lisbonne, éditeurs), Paris, mai 1912, p. 3 a.

5. Reading University Library - George Allen \& Unwin Collection, Ms 3282/FSC 40/292. Correspondance entre J. W. Swain et Cecil A. Reynolds, 14 octobre 1914 - 28 septembre 1915.

6. Reading University Library - George Allen \& Unwin Collection, Ms 3282/FSC 40/292. Lettre de J. W. Swain à Cecil A. Reynolds, Paris, 4 février 1915, f. 1. 
Graph. 1. Les deux moments de la première réception des Formes élémentaires
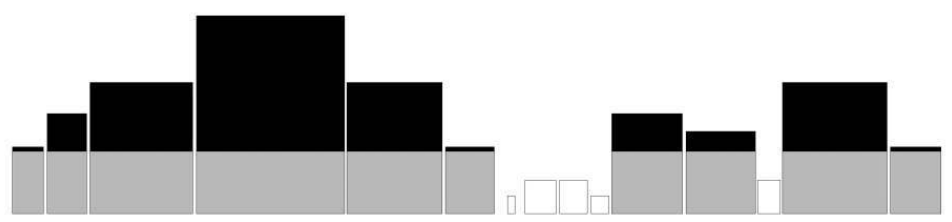

Nombre de recensions
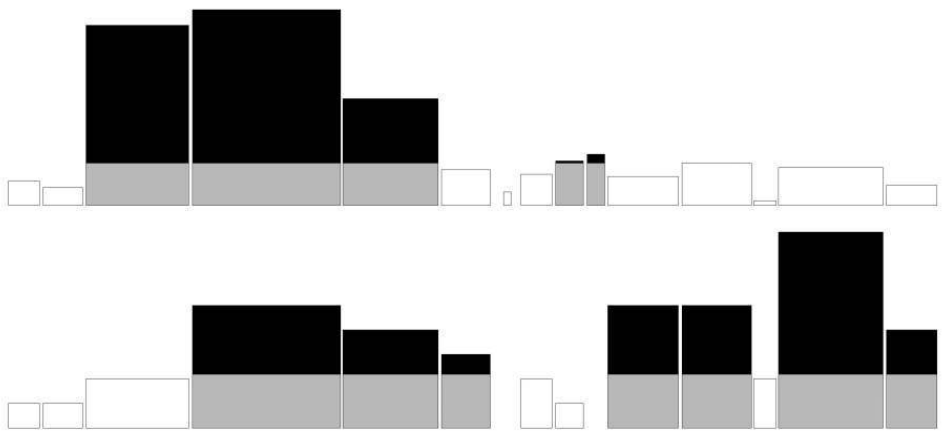

Taille des recensions
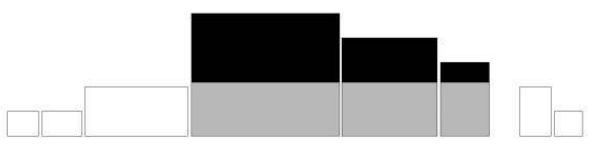

Nombre des recensions, $\mathrm{n}=74$. Volume total des recensions, $\mathrm{n}=1258338$ signes

Graph. 2. Les cadres disciplinaires de l'évaluation des Formes élémentaires: essai de classification

\section{Revues et journaux généralistes} Athenaeum (London) Deutsche Literaturzeitung für Kritik der internationalen Wissenschaft (Berlin)

Frankfurter Zeitung und Hondelsbiatt (Frankfort) La Grande Revue (Paris)

Le Correspondant (Paris)

LeTemps (Paris)

Literarisches Zentralblatt (Leipzig)

Mercure de France (Paris)

Potybiblion, revue bibliographique univesselie (Paris)

Revue critique des livres nouveoux (Paris)

Revue des idées. Sclence - philosophie - critique générale (Paris)

The American Year Book (New York)

The Annals of the American Academy (Philadelphia)

The Athenaeum (London)

The Evening Post (Melbourne)

The Trish Times (Dublin)

The Journal of Education, a Monthly Record and Review (London) The Nation (New York)

The New Statesman (London)

The Oultiook (New York)

The Saturday Review of Politics, literature and Art (London)

The Scotsman (Edinburgh)

The Times Literary Supplement (London)

\section{Sciences}

La Nature (Paris)

Scientia (Rivista di scienza) (Bologne

London /Paris / Leipzig)

\section{Revues et journaux confessionnels}

international Review of Mission (Geneva)

Le Christianisme Social (Paris)

Revue chrétienne (Paris)

Revue du Clergé français (Paris)

Revue pratique d'apologétique (Paris) [2]

The Biblical World (Chicago)

The Expository Times (Edinburgh)

\section{Revues et journaux spécialisés (disciplines)}

\section{Philosophie}

International Journal of Ethics (Philadelphia)

LAnnée phitosophique (Paris)

Revue de métaphysique et de morale (Paris) [2]

Revue philosophique de la Fronce et de létranger (Paris)

Rivista di Filasofia (Genova)

The Philosophical Review (New York/Lancaster) [2]

Philosophie et théologie

Review of Theology \& Philosophy (Edinburgh) Revue de théologie et de philosophie (Lausanne)

Revue des sciences philosophiques et théologiques (Kain)

The Hibbert Journal o Quarterly Review of Retigion Theology, and Philosophy (London)

\section{Théologie}

The American Sournal of Theology (Chicago) (New York) Theologische Literaturzeitung (Leipzig)

\section{Sociologie}

L'Année sociologique (Paris) [2]

Revue internationale de sociologie (Paris)

Rivista Italiana di Sociologia (Roma)

The American Journal of Sociology (Chicago) [2]

\section{Science sociale}

Athena, revue publice parl'EHES (Paris)

Bulletin de la Société internationale de Science

Sociale (Paris)

La Réforme sociale (Paris)

Le Musee Social - Annales (Paris)

Zeitschriff für Socialwissenschaft (Leipzig)
Histoire et archéologie

Revue archéologique (Paris)

Revue de l'histoire des religions (Paris)

Revue des études anciennes (Bordeaux)

Revue d'histoire et de littérature religieuse (Paris)

Revue historique (Paris)

Anthropologie

American Anthropologist (Washington) Man (London)

\section{Folkore}

Folk-Lore. Transactions of the Folk-Lore Society (London)

\section{Psychologie religieuse}

Journal of Religious $P_{\text {sychology, including its }}$ Anthropological and Sociological Aspects (Worcester)

\section{Medecine}

The American Journat of Nursing

(Philadelphia) 
Le public international visé est celui de la scène anthropologique alors dominée par les Britanniques et la figure tutélaire de James George Frazer. La liste des recensions témoigne enfin de la variété disciplinaire des revues et des rubriques sous lesquelles les Formes furent chroniquées et évaluées (Graph. 2).

Ce qu'il importe ici d'observer, c'est d'abord l'importance des publications généralistes qui ont été un des supports de diffusion de l'ouvrage à destination du grand public cultivé. Il faut ensuite noter la place des revues et journaux ouvertement confessionnels qui jouèrent un rôle charnière dans les discussions des thèses de Durkheim, notamment celles nouées au sein des revues plus spécialisées de philosophie ou de théologie qui sont particulièrement nombreuses : ces dernières représentent $23 \%$ des recensions, à comparer aux $33 \%$ des revues et journaux généralistes et aux $12 \%$ des revues confessionnelles. Moins nombreuses, celles-ci ont pourtant fait jeu égal si l'on veut bien considérer la taille des recensions publiées. Ainsi présenté, le corpus de la première réception apparaît très largement dominé par des publications dont la vocation et les ambitions sont, sinon universelles, du moins très larges, que l'on considère les publics visés ou les objets culturels qu'elles mettent en circulation. Reçue dans ce cadre particulièrement généraliste, la " sociologie religieuse » mise en œuvre par Durkheim dans les Formes gagna assurément en audience mais, évaluée dans ce même cadre, elle révéla tout aussi sûrement une de ses ambiguités : quel type de généralité ou de vérité, philosophique, scientifique et même, pourquoi pas, religieuse, visait son ouvrage ? La question, maintes fois posée par les recensions relevant de cette région la plus large de l'espace de réception, pointait cette incertitude. On y relevait abondamment les excès « métaphysiques » de l'ouvrage de Durkheim, on y dénonçait aussi, suivant un autre idiome, chez d'autres locuteurs, ses ambitions "théologiques ». La réception du côté des revues disciplinaires spécialisées, permet en outre de mettre en perspective ce que nous observons à l'échelle plus fine des rubriques sous lesquelles l'ouvrage de Durkheim a été recensé. Ce qui domine, c'est l'étroitesse de l'espace occupé par la sociologie, l'anthropologie et la science sociale. Un espace des disciplines là encore dominé par la philosophie, et secondairement par l'histoire, qui explique que la "sociologie religieuse » avancée par Durkheim relevait communément, si l'on s'en tient à la lettre des intitulés disciplinaires des rubriques sous lesquelles l'ouvrage fut effectivement recensé, des "Sciences philosophiques et religieuses ", de la "Philosophie de la religion ", de la «Philosophie religieuse » ou encore de la "Sociologie et histoire des religions ». Ce point révèle que, dans l'état des configurations savantes de l'époque, l'identité et le projet disciplinaire portés par les Formes n'étaient pas assurés, puisqu'ils demandaient des explications et suscitaient des discussions infinies qui travaillent presque chacune des recensions de l'ouvrage.

Plus généralement, d'un point de vue historique et sociologique, la valeur du corpus constitué sur la base de cette bibliographie tient au fait que les énoncés et les évaluations qui s'y trouvent rassemblés ont été produits in situ à propos 
d'un ouvrage qui se découvrait dans le vif de son actualité historique. Cela permet de donner un contenu spécifique à la notion de "première réception ", du vivant de Durkheim. En effet, même s'il fut longtemps annoncé et que l'introduction avait été livrée au public philosophique quelques années auparavant, l'ouvrage fut littéralement découvert, sans commentaires amont. Un ouvrage radicalement nouveau dont la signification, l'importance, les conséquences et les éventuels prolongements n'étaient pas alors connus de ses commentateurs : certains se sont risqués à des prophéties, plus ou moins réalisées. Perspicace, Salomon Reinach écrit au mois d'août 1912 à son ami Franz Cumont qu'il vient de lire " un chef-d'œuvre qui est appelé à exercer une très grande influence " ${ }^{7}$; un recenseur anonyme note que l' « ingénieuse théorie » de Durkheim sera «bien accueillie », ce qui ne fut pas précisément le cas. Célestin Bouglé, plus politique, conclut sa recension, la première que nous connaissions, en déclarant que " la lice est ouverte ». Nombreux sont ceux qui effectivement s’y précipitèrent.

Stéphane BACIOCCHI

Centre de Recherches Historiques Centre d'Anthropologie Religieuse Européenne

(EHESS-CNRS)

baciocch@ehess.fr

\section{Bibliographie}

Allen Nicholas J., Pickering William Stuart Frederick, Watts Miller William (eds), 1998, On Durkheim's Elementary forms of religious life, Londres/New York, Routledge, coll. "Routledge studies in social and political thought ", 10.

Coe George Albert, 1916, The Psychology of Religion, Chicago (Ill.), The University of Chicago Press.

FARgues Paul, 1913, "La religion d'après M. Durkheim », Revue chrétienne, publiée sous la direction de M. John Viénot. Recueil mensuel (Paris), 4érie, 60 (1), $1^{\mathrm{er}}$ mars, p. [255]-270 et $1^{\text {er }}$ mai, p. [426]-439.

FonsegRIVE George, 1915, "L'origine de la religion ", Le Correspondant. Religion Philosophie - Politique - Histoire - Sciences - Économie sociale - Voyages - Littérature Beaux-Arts (Paris), 87 (223), 10 avril, p. 121-149.

Fournier Marcel, 2007, "Aux origines de la vie religieuse », Émile Durkheim (18581917), Paris, Librairie Arthème Fayard, coll. "Histoire de la pensée ", p. 757-808.

GeHLke Charles Elmer, 1915, Emile Durkheim's Contributions to Sociological Theory, Submitted in partial fulfillment of the requirements for the degree of Doctor of philosophy in the Faculty of Political Science, New York, Columbia University.

Goddju Hans Petrus Marie, 1983, «De kerkelijke opponen van Emile Durkheim [Les opposants ecclésiastiques d'Émile Durkheim], Sociologisch Tijdschrift (Amsterdam), 10 (1), p. 147-171.

7. Academia Belgica (Rome) - Archives Franz Cumont, pi.5385, lettre de Salomon Reinach à " Mon cher ami ", Wiesbaden, [août 1912], f. 1v. 
Jordan Louis Henry, 1915, Comparative Religion. Its Adjuncts and Allies, Londres/ Edinburgh/Glasgow/New York/Toronto/Melbourne/Bombay, Humphrey Milford/ Oxford University Press.

Lukes Steven, 1988 [1973], "Durkheim and His Critics ", Émile Durkheim. His Life and Work. A Historical and Critical Study, $3^{\mathrm{e}}$ édition révisée, Londres, Peregrine Books, p. 6-529.

Morrison Ken, 2001, "The Disavowal of the Social in the American Reception of Durkheim ", Journal of Classical Sociology, 1 (1), p. 95-126.

NANDAN Yash, 1977, The Durkheimian school: a systematic and comprehensive bibliography, Westport (Conn.)/Londres, Greenwood press.

PICKERING William Stuart Frederick, 1975, Durkheim on Religion. A Selection of Readings with Bibliographies, Londres/Boston, Routledge and K. Paul.

-, 1984, Durkheim's Sociology of Religion. Themes and Theories, Londres, Routledge and K. Paul.

-, 2008, "The Response of Catholic and Protestant Thinkers to the Work of Émile Durkheim. With Special Reference to Les formes élémentaires ", Durkheimian Studies/ Études Durkheimiennes, 14, p. 59-93.

Pizarro Noël François, 2009, Du désaveu du social à la présentation nominaliste. Le mouvement de la réception de Durkheim (1893-1939), Thèse de doctorat en sociologie, Université du Québec à Montréal.

Rawls Anne Warfield, 1997, « Durkheim's Epistemology. The Initial Critique 1915-1924 », The Sociological Quarterly, 38 (1), Hiver, p. 111-145.

SEgER Imogen, 1957, Durkheim and his Critics on the Sociology of Religion, Foreword by Charles Y. Glock, Columbia University, Bureau of Applied Social Research, "Monograph Series ".

Simon Paul, 1918, Der Pragmatismus in der modernen französischen Philosophie, Münster in Westfalen, Druck der Westfälischen Vereinsdruckerei.

VAn Gennep Arnold, 1920, "Le livre de Durkheim. Son appréciation par Goldenweiser, Hartland, Loisy, Goblet d'Alviella, Bruno et Malinowski ", L'État actuel du problème totémique, Paris, éditions Ernest Leroux, p. 40-57. 


\section{Le corpus de la première réception}

Les deux parties de la bibliographie distinguent les recensions portant sur l'édition originale des Formes élémentaires (1912) de celles qui rendent compte de sa traduction anglo-américaine (1915). Nous y avons classé les entrées bibliographiques suivant l'ordre alphabétique des auteurs, dont le nom et les prénoms ont été restitués sous une forme complète. Les comptes rendus restés anonymes sont indiqués comme tels (Anon.) ; ceux pour lesquels l'anonymat a pu être levé sont signalés par la mise entre crochets du nom de leur auteur. L'énoncé des entrées bibliographiques, notamment le «titre » des recensions - qui souvent n'en possèdent pas - a été formé en suivant une règle simple : lorsqu'elle existe, la rubrique bibliographique sous laquelle l'ouvrage de Durkheim a été recensé est toujours indiquée, suivie de l'éventuel titre de la recension. La mention bibliographique de l'ouvrage recensé a été omise. Elle fait l'objet d'une description précise en tête de chacune des parties. Enfin, le titre des périodiques est donné aussi complètement que nécessaire. Il est suivi de l'indication de son lieu d'édition suivant sa graphie vernaculaire.

La publication des Formes élémentaires fut annoncée en mai 1912 par une brève notice de l'éditeur que nous publions ici en raison de sa rareté et de son intérêt documentaire :

\section{Les Formes primitives de la Vie religieuse. Le système totémique en Australie} par É. Durkheim, professeur à la Sorbonne

1 vol. in 8 de la Bibliothèque de Philosophie contemporaine... $15 \mathrm{fr}$ (Paraîtra le 15 juin)

L'objet de ce livre est d'atteindre, à travers un groupe déterminé de religions, les formes élémentaires de la pensée et de la pratique religieuse. Les religions étudiées sont celles que pratiquent les tribus australiennes. Il n'en est pas de plus primitives ni de plus simples et c'est ce qui explique le choix de l'auteur, [sic] on a des chances d'y découvrir les éléments fondamentaux de la vie religieuse et les causes dont ces éléments dépendent.

Comme toute religion est une cosmogonie, on ne peut analyser la pensée religieuse sans rencontrer sur sa route les notions qui dominent la pensée logique - notions de temps, d'espace, de classe, de force et de cause, de personnalité. L'auteur montre comment ces idées sont nées dans la religion et de quelles causes sociales elles résultent. La sociologie religieuse apporte ainsi une importante contribution à la théorie de la connaissance ${ }^{8}$.

I

Les Formes élémentaires de la vie religieuse. Le Système totémique en Australie, par Émile Durkheim, professeur à la Faculté des lettres de l'Université de Paris, Paris, lib. Félix Alcan, "Travaux de L'Année sociologique " - "Bibliothèque de philosophie contemporaine ", [juin] 1912, 647 p. avec une carte hors texte. Imprimé à Évreux sur les presses de P. Hérissey, l'ouvrage est signalé parmi les livres qui "Viennent de paraître " dans le "Feuille-

8. Bulletin des publications nouvelles de la librairie Félix Alcan (Félix Alcan et R. Lisbonne, éditeurs), Paris, mai 1912, p. 3a. 
ton " de la Bibliographie de la France du 14 juin (vol. 101, $2^{\mathrm{e}}$ série, 24, p. 2046) puis dans le numéro du journal Le Temps daté du 23 juin 1912. Vendu 10 francs, son tirage est de 1650 exemplaires.

Anon., "Revue de livres ", Revue chrétienne (Paris), 4e série, 59 (2), juilletaoût 1912, p. 752b-753a.

Anon., "Bibliographie ", La Nature. Revue des sciences et de leurs applications aux arts et à l'industrie (Paris), $\mathrm{n}^{\circ} 2056$ (supplément), 19 octobre 1912, p. 167.

Anon., "Recensioni e cenni ", Rivista di Filosofia, organo della Società Filosofica Italiana (Genova), 4 (4), LuglioOttobre 1912, p. 543.

ANON., « Memento Bibliographique Méthodique - Sciences philosophiques et religieuses ", La Grande Revue (Paris), 16 (22), 25 novembre 1912, p. 439.

Anon., "Livres nouveaux", Revue de métaphysique et de morale (Paris), 21-3 (supplément), mars 1913, p. 1-3.

Belot Gustave, "Une Théorie nouvelle de la religion", Revue Philosophique de la France et de l'étranger (Paris), 75 (4), avril 1913, p. 329-379.

Benrubi Isaak, "Emile Durkheims Religiöse Soziologie ", Frankfurter Zeitung und Handelsblatt (Frankfort), 53, 22. Februar 1914, p. 7a-8c.

Bouglé Célestin, "Variétés philosophiques Une nouvelle philosophie de la religion ", Le Temps (Paris), 42 (18644), samedi 20 juillet 1912, p. 2f-3b.

BREYSIG Kurt, "Theologie. Religionswissenschaft ", Literarisches Zentralblatt für Deutschland (Leipzig), 65 (48), 28. November 1914, p. 1481-1484.

BRUNO Alessandro, "Rassegne Analitiche L'origine della religione e il totemismo ", Rivista Italiana di Sociologia (Roma), 8 (5-6), Settembre-Dicembre 1914, p. 749-754.

Chatterton-Hill George, «Recensioni Comptes rendus - Referate - Book
Reviews ", "Scientia» (Rivista di scienza). Organo internazionale di sintesi scientifica (Bologne-London/Paris/ Leipzig), 13 (27-1), 1913, p. 126-129.

ClÉMEnT Henry, "Mélanges et notices, III. - Religion, morale et sociologie", La Réforme sociale, Bulletin de la Société d'économie sociale et des Unions de la paix sociale fondées par P.-F. Le Play (Paris), 60 (117-118), Livraison du $1^{\text {er }}-16$ novembre 1915 , p. 430-434.

Descamps Paul, "Le totémisme chez les indigènes de l'Australie ", Bulletin de la Société Internationale de Science Sociale (Paris), 27 (96), septembre 1912, p. 105b-110b.

Durkheim Émile, «Analyses - Sociologie générale, IV. - Conditions sociologiques de la connaissance ", L'Année sociologique (Paris), tome XII, (19091912), 1913, p. 33-37.

FArgues Paul, «Études sociologiques : Le point de vue sociologique de M. Durkheim ", Le Christianisme Social. Revue mensuelle d'étude \& d'action (Paris), 26 (4), avril 1913, p. 234-246.

Fargues Paul, «La religion d'après M. Durkheim ", Revue chrétienne (Paris), $4^{\mathrm{e}}$ série, 60 (1), $1^{\mathrm{er}}$ mars 1913, p. 255-270 et, $1^{\text {er }}$ mai 1913 , p. 426439.

Fonsegrive George, "L'origine de la religion ", Le Correspondant (Paris), 87 (223), 10 avril 1915, p. 121-149.

Goblet D’Alviella Eugène, "Mélanges et documents - La Sociologie de M. Durkheim et l'Histoire des religions ", Revue de l'histoire des religions (Paris), 67, 1913, p. 192-221.

GoldENWEISER Alexander Aleksandrovich, «Book Reviews - Methods and Principles ", American Anthropologist (Washington), n.s. 17 (4), OctoberDecember 1915, p. 719-735. 
Habert Paul Onésisme, "La religion primitive d'après l'école sociologique", Revue $d u$ Clergé Français (Paris), 75 (451), $1^{\text {er }}$ septembre 1913 , p. 513543.

Hartland Edwin Sidney, «Review - Australia: Totemism », Man, a Monthly Record of Anthropological Science (London), 13 (5), [May] 1913, p. 91-96.

HøFFdING Harald, 1914, «Études critiques - Les formes élémentaires de la vie religieuse", Revue de métaphysique et de morale (Paris), 22 (6), [décembre] 1914, p. 828-848.

Jullian Camille, "Bibliographie ", Revue des études anciennes (Bordeaux), 16 (2), avril-juin 1914, p. 244-250.

KING Irving, "Reviews of Books ", The Philosophical Review (New York/ Lancaster), 22 (4), July 1913, p. 431434.

Kodisowa Józefa Fabianna, "Sprawozdania ", Ruch Filozoficzny (Lwów), 3 (5), 15 maja 1913, p. 111a-112a.

LALANDE André, "Philosophy in France in 1912 ", The Philosophical Review (New York/Lancaster), 22 (4), July 1913, p. 357-374.

LANG Andrew, "The Origin of Belief in a God ", The Athenaeum (London), 4423, August 3, 1912, p. 119b-c.

LANG Andrew, "Formes élémentaires de la vie religieuse ", 1913, Review of Theology \& Philosophy (Edinburgh), 8, July 1912-June 1913, p. 115-120.

LEMONNYER Antoine, "Bulletin de science des religions. I. Généralités. Origine de la religion ", Revue des sciences philosophiques et théologiques (Kain), 7 (4), 20 juillet 1913, p. 535-537.

LoISY Alfred, "Sociologie et religion", Revue d'histoire et de littérature religieuses (Paris), 4 (1), janvier-février 1913, p. 45-76.

MaInAgE Thomas-Lucien, «Chronique d'histoire des religions ", Revue pratique d'apologétique (Paris), 17 (200), 15 janvier 1914, p. 621-625.
Maisonneuve Louis, "Philosophie-MoraleSociologie-Esthétique ", Polybiblion, revue bibliographique universelle (Paris), 125 (25), novembre 1912, p. 393-394.

MALINOwSKI Bronisław Kasper, "Reviews ", Folk-Lore. Transactions of the FolkLore Society (London), 24 (4), December 1913, p. 525-531.

MARTIN Eleuthère, "Chronique religieuse Les formes élémentaires de la vie religieuse", Athena, revue publiée par l'École des hautes études en sciences sociales (Paris), 19, octobre-novembre 1912, p. 344-348.

MARTIN-SAINT-LÉON Étienne, «Bulletin bibliographique ", Le Musée Social Annales, revue mensuelle (Paris), 7, juillet 1913, p. 230.

Matisse Madeleine, "L'origine des religions ", Revue des idées. Science philosophie - critique générale, 9 (99), 15 décembre 1912, p. 251-281.

Maunier René, « Revue des livres, I. - Histoire des religions ", Revue internationale de sociologie (Paris), 21 (4), avril 1913, p. 276

Mauss Marcel, Durkheim Émile, "Analyses - Sociologie religieuse, II. - Systèmes religieux des sociétés inférieures. A. - Le système totémique. ", L'Année sociologique (Paris), tome XII (19091912), 1913, p. 91-98.

MAYer Emil Walter, "Durkheim, Les Formes élémentaires de la vie religieuse ", Theologische Literaturzeitung (Leipzig), 38 (18), 30. August 1913, p. 547-549.

Michelet Georges, "Chronique de Philosophie - Principaux courants actuels en philosophie religieuse ", Revue pratique d'apologétique (Paris), 16 (190), 15 août 1913, p. 778-797.

MiéviLle Henri-Louis, "Revue générale Philosophie de la religion ", Revue de théologie et de philosophie (Lausanne), n.s., 1, janvier 1913, p. 82-91. 
Oltramare Paul, "Comptes rendus critiques", Revue historique (Paris), 38 (113), mai-août 1913, p. 365-367.

PILlON François, "Bibliographie philosophique française de l'année 1912, II. - Morale, philosophie et histoire religieuse ", L'Année philosophique (Paris), 23, 1913, p. 229-232.

Pradel Félix, "Analyses et comptes rendus, II. - Philosophie religieuse", Revue de philosophie (Paris), 22 (2), février 1913, p. 317-321.

Preuss Konrad Theodor, "Theologie und Kirchenwesen - Referate ", Deutsche Literaturzeitung für Kritik der internationalen Wissenschaft (Berlin), 35 (3), 17 Januar 1914, p. 147-149.

ReINACH Salomon, "Bibliographie ", Revue archéologique (Paris), 4 (20), juilletaoût 1912, p. 182.

ReINACH Salomon, "Une étude sur les religions primitives", Revue critique des livres nouveaux (Paris), n.s. 7 (8), 15 octobre 1912, p. 153-154.

VAn Gennep Arnold, "Revue de la quinzaine - Ethnographie, folklore", Mercure de France (Paris), 101 (374), 16 janvier 1913, p. 389-391.

VIERKANDT Alfred, "Buchbesprechungen ", Zeitschrift für Socialwissenschaft (Leipzig), IV (6), 4, Juni 1913 p. 439440.

Wallis Wilson Dallam, « Durkheim's View of Religion ", Journal of Religious Psychology, including its Anthropological and Sociological Aspects (Worcester, Mass.) 7 (2), November 1914, p. 252267.

Webster Hutton, "Reviews ", The American Journal of Sociology (Chicago), 18 (6), May 1913, p. 843-846.

\section{II}

The Elementary Forms of the Religious Life. A Study in Religious Sociology. By Émile Durkheim, Professor of the Faculty of Letters at the University of Paris. Translated from the French by Joseph Ward
Swain, M.A., London: George Allen \& Unwin Ltd, [September] 1915. Pp. xii, 456 includes an index. Imprimé à Plymouth sur les presses de William Brendon \& Son, l'ouvrage est signalé comme "Just out " dans la page publicitaire de la Saturday review of politics, literature, sience and art du 16 octobre 1915 (vol. 120, no 3129, p. 381). Vendu 15 shillings, son tirage est de 1000 exemplaires dont 250 sont à destination des États-Unis où l'ouvrage est diffusé depuis New York par The Macmillan Company au prix de 4 dollars.

ADAMS George Plimpton, " Book Reviews ", The Harvard Theological Review (New York), 10 (2), April 1917, p. 201-204.

AnON., "The Bookman ", The Evening Post (Melbourne), 90 (147), 18 December 1915 , p. 16.

ANON., "Books of the Week - Religious Sociology. ", The Irish Times (Dublin), October 1, 1915, p. 7b.

AnON., "New Books", The Scotsman (Edinburgh), October 28, 1915, p. 2.

Anon., "Science", The Athenaeum (London), 4595, November 20, 1915, p. 370 .

AnON., "Latest Books ", The Saturday Review of Politics, Literature and Art (London), 120 (3139), 25 December 1915, p. 616.

ANON., "Review and Minor Notices", The Journal of Education, a Monthly Record and Review (London), 48 (560), March 1, 1916, p. 151-152.

ANON., "Book Notices ", The Biblical World (Chicago), 47 (6), June 1916, p. 413.

ANON., "Recent Sociological Studies ", The Outlook (New York), 113, August 2, 1916, p. 808-809.

ANON., "Religious Sociology ", The Expository Times (Edinburgh), 27, October 1915, p. 77b-78b. 
AnOn., "The Totem and Society ", The Nation (New York), 103 (2663), July 1916, p. 39-40.

BECKERSON William Townley, "Notices of Books ", The Irish Church Quarterly (Dublin), 9 (35), July 1916, p. 245 247.

BoIs Henri, "A Sociological View of Religion ", The International Review of Missions (Geneva), 5 (3), July 1916, p. 449-460.

CAMERon M. Ednah, "Book Reviews", The American Journal of Nursing (Philadelphia), 16 (12), September 1916, p. 1248-1249.

[ELIOT Thomas Stearns], "Durkheim ", Westminster Gazette, (London), 48 (7235), August 19, 1916, p. 2 et Saturday Westminster Gazette (7235), August 19, 1916, p. 14.

[Haddon Alfred Cort], "The Roots of Religion ", The Times Literary Supplement (London), Thursday, December 23, 1915, p. 488a-b.

HARrison Jane Ellen, "Miscellany Durkheim ", The New Statesman, a Weekly Review of Politics and Literature (London), 6 (133), October 23, 1915, p. 62b-64a.
JoHnston George Alexander, "Book Reviews ", International Journal of Ethics (Philadelphia), 26 (2), January 1916, p. 303-304.

LEWIS Albert Buell, "Recent theological literature - A Study in Religious Sociology ", The American Journal of Theology (Chicago), 21 (1), January 1917, p. 143-148.

Lichtenberger James Pendleton, "Book Department - Sociology and Modern Social Problems ", The Annals of the American Academy (Philadelphia), 66-68, July 1916, p. 263.

Moffat James, "Survey of Recent Philosophical and Theological Literature Theology ", The Hibbert Journal, a Quarterly Review of Religion, Theology, and Philosophy (London), 14 (2), March 1916, p. 436-437.

PERRY Ralph Barton, "Philosophy of Religion ", The American Year Book. A Record of Events and Progress. 1916, New York/London, D. Appleton and Company, 1917, p. 672b.

Weatherly Ulysses Grant, "Reviews", The American Journal of Sociology (Chicago), 22 (4), January 1917, p. 561563. 